\title{
InAs Planar Nanowire Gate-All-Around MOSFETs on GaAs Substrates by Selective Lateral Epitaxy
}

\author{
Chen Zhang, Student Member, IEEE, Wonsik Choi, Parsian K. Mohseni, Member, IEEE, \\ and Xiuling Li, Senior Member, IEEE
}

\begin{abstract}
High indium content III-V materials are one of the most promising candidates for beyond Si CMOS technologies. We present InAs planar nanowire (NW) MOSFETs grown directly on a semi-insulating GaAs (100) substrate by the selective lateral epitaxy (SLE) method via the metal-seeded planar vapor-liquid-solid mechanism. Despite a $\sim 7 \%$ lattice mismatch, in-plane and self-aligned single-crystal InAs NWs are grown epitaxially on GaAs. Such heterogeneous SLE provides a potential solution for the integration of different channel materials on one substrate. Gate-all-around MOSFET devices are fabricated by releasing the NW channel from the substrate through a combination of digital etching and selective etching processes. The device with a NW width of $30 \mathrm{~nm}$ and gate length of $350 \mathrm{~nm}$ shows an $I_{\mathrm{ON}} / I_{\mathrm{OFF}}$ ratio of $10^{4}$ and a peak transconductance of $220 \mathrm{mS} / \mathrm{mm}$ at $V_{\mathrm{ds}}=0.5 \mathrm{~V}$.
\end{abstract}

Index Terms-III-V MOSFETs, InAs, VLS growth, nanowire, selective lateral epitaxy.

\section{INTRODUCTION}

III -V MATERIALS, especially InGaAs with high In-content (In\%), are promising candidates for future low-power logic applications due to their excellent electron mobility [1]. State-of-the-art InGaAs channel MOSFETs are mostly based on InGaAs closely lattice-matched to InP substrates [2], or extremely thin-body InAs films [3]-[5]. For in-plane nanowire (NW) channels fabricated by etching a thin film layer, it is difficult to achieve a high In\% due to the lattice mismatch with respect to a common growth substrate, such as GaAs or InP. The bottom-up self-assembled vaporliquid-solid (VLS) method, which utilizes a metal particle (usually $\mathrm{Au}$ ) to seed and direct the NW growth [6], [7] can grow high-quality NWs on highly lattice-mismatched substrates as nanostructures can accommodate more strain than standard thin films [8], [9]. This provides a promising solution for the formation of true 3D channels with high In\%, and the heterogeneous integration of $\mathrm{n}$-channel and p-channel materials, such as InAs and GaSb, on a single substrate. However, one complication of such a self-assembly method is that the NWs mostly grow out of the substrate

Manuscript received April 14, 2015; revised April 27, 2015; accepted May 1, 2015. Date of publication May 5, 2015; date of current version June 24, 2015. This work was supported by the National Science Foundation ECCS Award \#1001928 (CZ, XL) and DMR Award \#1006581 (WC, PM, XL). The review of this letter was arranged by Editor M. Passlack.

The authors are with the Department of Electrical and Computer Engineering, University of Illinois, Urbana, IL 61822 USA (e-mail: xiuling@illinois.edu).

Color versions of one or more of the figures in this letter are available online at http://ieeexplore.ieee.org.

Digital Object Identifier 10.1109/LED.2015.2429680
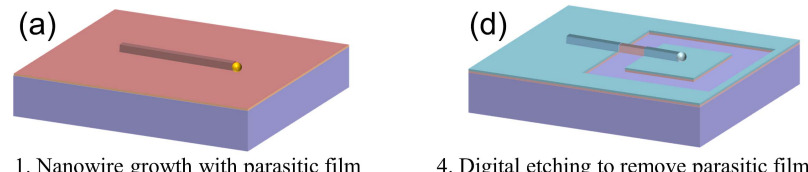

1. Nanowire growth with parasitic film

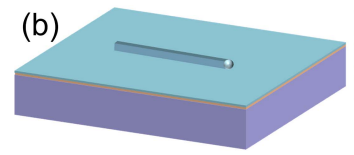

2. $\mathrm{ALD} \mathrm{Al}_{2} \mathrm{O}_{3}$ as etching mask

(c)

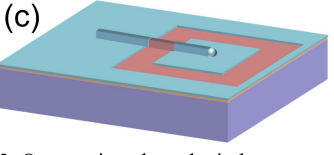

3. Open a ring-shaped window
4. Digital etching to remove parasitic film
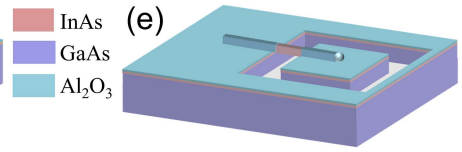

5. Selectively etch GaAs to release channel

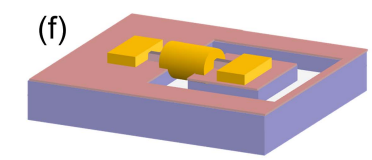

6. Deposition of gate oxide and contacts
Fig. 1. Schematic diagram illustrating the key steps for the planar NW GAA MOSFET fabrication.

surface in the $<111>$ directions and, therefore, much more complicated fabrication processes are required than planar devices [10]. Furthermore, the vertical architecture inherently incurs more parasitic capacitance due to contact pad overlapping, which affects the speed performance [10]. while vertical NW transistors will continue to be developed and assessed by researchers, we have shown that VLS growth can take place in a planar fashion where the NWs grow laterally and are self-aligned along certain in-plane crystal directions [6], [7]. Therefore, we call this particular VLS method selective lateral epitaxy (SLE), where the selectivity is provided by the metal seed particles and the epitaxial relationship to the substrate ensures high crystal quality. The planar NWs are completely compatible with standard planar processing technology. Many types of devices, including MESFETs [11], HEMTs [12] and MOSFETs [13], and simple circuits [14] have been demonstrated to show good performance by homogenous SLE, namely, GaAs planar NWs on GaAs substrates.

In this letter, we demonstrate for the first time a heterogeneous-SLE InAs NW gate-all-around (GAA) MOSFET on GaAs (100). The devices are realized by releasing the NW channel from the substrate using a combination of digital and selective etching, while keeping the source and drain in plane. This work demonstrates the feasibility of InAs-based low power FETs and future heterogeneous device integration using SLE.

\section{EXPERIMENTS}

Fig. 1 shows the process flow for the GAA MOSFET device fabrication, starting from planar InAs NWs directly 

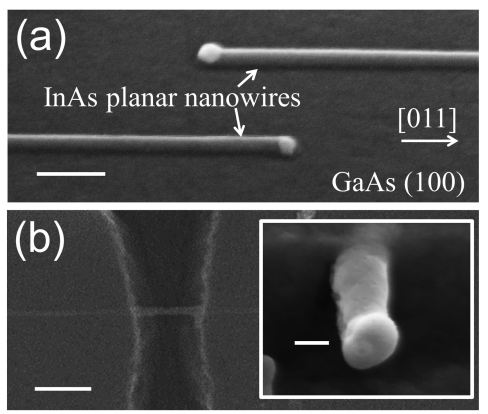

Fig. 2. (a) $\mathrm{A} 45^{\circ}$ tilted SEM image of two as-grown InAs planar NWs on GaAs (100). Au dots are visible at the tips of the NWs. The scale bar is $100 \mathrm{~nm}$. (b) SEM image showing a NW hanging over the trench after releasing ( $300 \mathrm{~nm}$ scale bar). The inset $(50 \mathrm{~nm}$ scale bar) shows the cross section of a fabricated NW device with conformal gate metal coating.

grown on GaAs (100) semi-insulating (SI) substrates seeded by colloidal Au nanoparticles. The growth was carried out in a metalorganic chemical vapor deposition (MOCVD) system at $340{ }^{\circ} \mathrm{C}$ with trimethylindium (TMIn) and arsine $\left(\mathrm{AsH}_{3}\right)$ as precursors, without intentional doping. A typical growth rate was $\sim 360 \mathrm{~nm} / \mathrm{min}$. A thin parasitic InAs film (with thickness on the order of a few nanometers) covering the entire sample surface was simultaneously formed during growth via the competing vapor-solid process, as illustrated in Fig. 1(a). The parasitic thin film, highly conductive according to the substrate current measurement, should be beneficial in reducing S/D resistance and can be readily removed in the channel region by digital etching [4], [5], as will be discussed later. Note that the parasitic thin film growth is much more sensitive to the growth temperature than the VLS NW growth; thus, can be reduced by lowering the temperature. Fig. 2(a) shows a SEM image of as-grown planar InAs NWs (width $\sim 25 \mathrm{~nm}$ ) selfaligned along either [011] or [0-1-1] direction, with Au seeds visible at the NW tips. We have found planar NWs grow along the projections of out-of-plane $<111>$ directions on the substrate surface [11] $(<111>$ B for GaAs planar NWs [11] and $<111>$ A for InAs [7]). Therefore, unidirectional growth, which is desired for high-density device integration, can be achieved on a (110) substrate because there is only one outof-plane $<111>\mathrm{B}$ or $<111>\mathrm{A}$ direction [11]. EDXS analysis has confirmed the NW to be InAs with no Ga detectable [7]. High-resolution TEM analysis can be found in [7], where the heterogeneous InAs planar NWs (with a Zincblende structure) are shown to be epitaxial to the GaAs substrate and free of stacking faults. NWs larger than $30 \mathrm{~nm}$ tend to form a zigzag trajectory on the substrate, presumably due to the strain effect.

To fabricate the MOSFET device, 12-nm $\mathrm{Al}_{2} \mathrm{O}_{3}$ was first deposited by ALD to be the etching mask for subsequent steps (Fig. 1(b)). A ring-shaped window was then opened on $\mathrm{Al}_{2} \mathrm{O}_{3}$ by e-beam lithography and $\mathrm{BOE}$ etching (Fig. 1(c)). In order to release the InAs NW from the underlying GaAs substrate, we removed the exposed InAs parasitic film (Fig. 1(d)) by digital etching [4], [5], where oxidation (at room temperature in a UV ozone cleaner from BioForce Nanoscience, Inc.) and oxide removal etching (by $1: 1 \mathrm{HCl}: \mathrm{H}_{2} \mathrm{O}$ solution) were

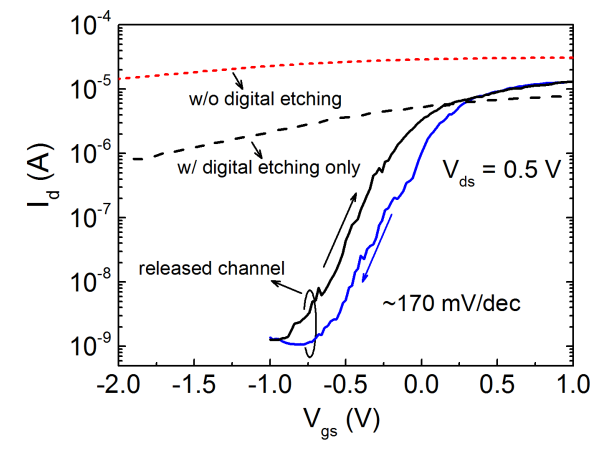

Fig. 3. Log-scale transfer characteristics of the InAs planar NW MOSFETs. Hysteresis could be due to the NW/oxide interface traps and/or mobile charges in the oxide.

performed alternately to etch III-Vs at $\sim 1 \mathrm{~nm}$ per cycle. Two cycles of digital etching were sufficient for full removal of the parasitic film so that the underlying GaAs could be exposed. A trench was then formed on GaAs to release the center portion of the NW by selectively etching GaAs against InAs using $\mathrm{NH}_{4} \mathrm{OH}: \mathrm{H}_{2} \mathrm{O}_{2}: \mathrm{H}_{2} \mathrm{O}$ (1:1:80) [15], similar to the release process of top-down InGaAs NW MOSFETs [2]. Fig. 2(b) shows a SEM image of a NW after releasing. 6-nm $\mathrm{Al}_{2} \mathrm{O}_{3}$ (EOT $=3 \mathrm{~nm}$ ) was then deposited by ALD at $220{ }^{\circ} \mathrm{C}$ as the gate oxide, followed by the $\mathrm{Ni} / \mathrm{Au}$ gate metal deposition by sputtering to provide conformal gate contact, as shown previously [16]. The inset SEM image of Fig. 2(b) shows the cross-section (after milling by focused ion beam (FIB)) of a fabricated device with the gate metal covering all around the NW. Finally, Ni/Au was evaporated for source and drain contacts after $\mathrm{Al}_{2} \mathrm{O}_{3}$ is removed on S/D region. No annealing was performed in the fabrication process. Although the current process leaves a large area of substrate covered by the parasitic film (Fig. 1(f)), which is undesired for circuit applications, in principle we can keep only the parasitic film in the S/D region by patterning.

\section{RESUlts AND Discussions}

Fig. 3 shows the log-scale transfer curves $\left(V_{d s}=0.5 \mathrm{~V}\right)$ of InAs NW MOSFETs with different fabrication schemes. The NW width, $d$, of these devices is $\sim 30 \mathrm{~nm}$. The device fabricated without digital etching and NW releasing (i.e., using as-grown NWs) showed very poor gate control (red, short-dashed curve). The as-grown substrate surface leakage current was measured to be 1-2 $\mu \mathrm{A}$ from two contacts not connected by a NW. This leakage path was removed by applying the digital etch treatment. The device with digital etching (applied to the entire substrate) showed some reduction of leakage current at $V_{g s}=-2 \mathrm{~V}$ (black, long-dashed curve). Significant reduction of off-state leakage was observed only after releasing the NW channel. Shown in Fig. 3 by the solid curves, the $I_{o n} / I_{\text {off }}$ ratio is as high as $10^{4}$ as $V_{g s}$ varies from $-1 \mathrm{~V}$ to $1 \mathrm{~V}$, with the subthreshold swing $(S S)$ measured from the positive-sweep curve $\left(V_{g s}=-0.6 \mathrm{~V}\right.$ to $\left.-0.4 \mathrm{~V}\right)$ to be $170 \mathrm{mV} / \mathrm{dec}$. This suggests the main leakage was induced by the bottom interface between InAs and GaAs. We speculate certain interface defects pin the 

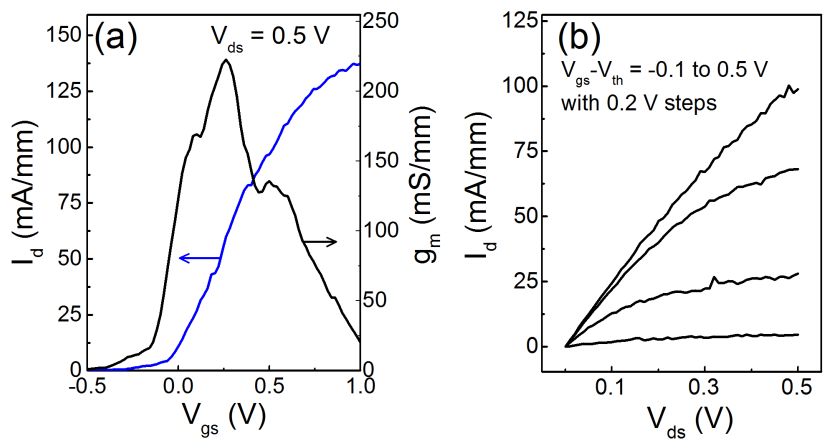

Fig. 4. (a) Transfer and transconductance curves of the GAA device with $d=30 \mathrm{~nm}$ an $L_{g}=350 \mathrm{~nm}$. (b) Typical output curves of the device with the same dimensions. All numbers are normalized by $\pi d$.

Fermi level of InAs close to its conduction band so that the device could not be turned off completely. Note that we define the gate length, $L_{g}$, to be the trench width (350 $\mathrm{nm}$ in Fig. 2(b)), because the non-released gated portion cannot be well modulated. The DIBL of the devices is $\sim 0.3 \mathrm{~V} / \mathrm{V}$. The on-state transfer curve and typical output characteristics of the GAA device are shown in Fig. 4. The threshold voltage is $\sim 0 \mathrm{~V}$. The device shows a peak extrinsic transconductance $\left(g_{m}\right)$ of $220 \mathrm{mS} / \mathrm{mm}$ at $V_{d s}=0.5 \mathrm{~V}$. The $R_{o n}$ is measured to be $4.1 \Omega \cdot \mathrm{mm}$ at $V_{o v}=0.5 \mathrm{~V}$. Both are normalized by $\pi d$.

The $S S$ and $g_{m}$ reported here are decent compared to other III-V NW devices summarized in [17], but are not as good as the state-of-the-art vertically grown VLS InAs NW device [18] and top-down InGaAs in-plane NW devices [2]. However, we note our device has a thick EOT $(3 \mathrm{~nm})$ and long $L_{g}(350 \mathrm{~nm})$ while both the state-of-the-art works above have an EOT of $\sim 1 \mathrm{~nm}$ and a much shorter $L_{g}$. We estimate the interface trap density $D_{i t}$ from $S S$ by $S S=\left(1+q \pi d D_{i t} / C_{o x}\right) \cdot 60 \mathrm{mV} / \mathrm{dec}$, where $q$ is the electron charge and $C_{O x}$ is the oxide capacitance per unit length. Thus, $D_{i t}$ is $1.6 \times 10^{13} \mathrm{~cm}^{-2} \mathrm{eV}^{-1}$. We also extract the fieldeffect electron mobility, $\mu_{F E}$, using the peak $g_{m}$ measured at $V_{d s}=0.05 \mathrm{~V}$. The gate capacitance was simulated by Nextnano [19] and estimated to be $1 \mathrm{pF} / \mathrm{cm}$ at the peak $g_{m}$. $D_{\text {it }}\left(1.6 \times 10^{13} \mathrm{~cm}^{-2} \mathrm{eV}^{-1}\right)$ was added to simulate the C-V stretch-out. The $\mu_{F E}$ is estimated to be $2730 \mathrm{~cm}^{2} / \mathrm{V} \cdot \mathrm{s}$, which appears to be lower than those reported in [20]. We believe by scaling down $L_{g}$, EOT and contact resistance, the device performance can be further improved.

In summary, we have presented experimental results of InAs planar NW GAA MOSFETs grown by SLE on highlymismatched GaAs substrates. Future studies will involve yield improvement and growth of planar p-channel (for example, $\mathrm{GaSb}$ ) NWs. One way to integrate $\mathrm{p}$ - and n-channel NWs for CMOS is through multi-level planar VLS method, with the NWs from the first level of growth masked before patterning seed particles for the second level growth of a different material. Fabrication of lateral $\mathrm{p}-\mathrm{n}$ heterostructures with only one level of seed particle patterning is also possible.
In addition, we note that using $\mathrm{Au}$ seeds is not desired for integration with $\mathrm{Si}$ CMOS. VLS growth of InAs NWs by CMOS compatible metal seeds, such as $\mathrm{Ni}$, has already been demonstrated [20]. SLE with a non-Au seed should be feasible and will be pursued in our future research.

\section{ACKNOWLEDGMENT}

The authors would like to thank M. W. Si and P. D. Ye for valuable discussions.

\section{REFERENCES}

[1] J. A. del Alamo, "Nanometre-scale electronics with III-V compound semiconductors," Nature, vol. 479, no. 7373, pp. 317-323, Nov. 2011.

[2] J. J. Gu et al., "20-80 nm channel length InGaAs gate-all-around nanowire MOSFETs with EOT $=1.2 \mathrm{~nm}$ and lowest $\mathrm{SS}=63 \mathrm{mV} / \mathrm{dec}$," in IEDM Tech. Dig., Dec. 2012, pp. 633-636.

[3] S. W. Chang et al., "InAs N-MOSFETs with record performance of $\mathrm{I}_{\mathrm{on}}=600 \mu \mathrm{A} / \mu \mathrm{m}$ at $\mathrm{I}_{\mathrm{off}}=100 \mathrm{nA} / \mu \mathrm{m}\left(\mathrm{V}_{\mathrm{d}}=0.5 \mathrm{~V}\right)$," in IEDM Tech. Dig., Dec. 2013, pp. 417-420.

[4] J. Lin et al., "A new self-aligned quantum-well MOSFET architecture fabricated by a scalable tight-pitch process," in IEDM Tech. Dig. Dec. 2013, pp. 421-424.

[5] S. Lee et al., "Record $\mathrm{I}_{\mathrm{on}}\left(0.50 \mathrm{~mA} / \mu \mathrm{m}\right.$ at $\mathrm{V}_{\mathrm{DD}}=0.5 \mathrm{~V}$ and $\left.\mathrm{I}_{\mathrm{off}}=100 \mathrm{nA} / \mu \mathrm{m}\right) 25 \mathrm{~nm}$-gate-length $\mathrm{ZrO}_{2} /$ InAs/InAlAs MOSFETs," in Symp. VLSI Technol. Dig. Tech. Papers, 2014, pp. 1-2.

[6] S. A. Fortuna et al., "Planar GaAs nanowires on GaAs (100) substrates: Self-aligned, nearly twin-defect free, and transfer-printable," Nano Lett., vol. 8, no. 12, pp. 4421-4427, Dec. 2008.

[7] C. Zhang et al., "Site-controlled VLS growth of planar nanowires: Yield and mechanism," Nano Lett., vol. 14, no. 12, pp. 6836-6841, Dec. 2014.

[8] L. C. Chuang et al., "Critical diameter for III-V nanowires grown on lattice-mismatched substrates," Appl. Phys. Lett., vol. 90, no. 4, p. $043115,2007$.

[9] P. K. Mohseni et al., "Monolithic III-V nanowire solar cells on graphene via direct van der Waals epitaxy," Adv. Mater., vol. 26, no. 22, pp. 3755-3760, Jun. 2014.

[10] M. Egard et al., "Vertical InAs nanowire wrap gate transistors with $f_{t}>7 \mathrm{GHz}$ and $f_{\max }>20 \mathrm{GHz}$," Nano Lett., vol. 10, no. 3, pp. 809-812, Mar. 2010.

[11] R. Dowdy et al., "Realization of unidirectional planar GaAs nanowires on GaAs (110) substrates," IEEE Electron Device Lett., vol. 33, no. 4, pp. 522-524, Apr. 2012.

[12] X. Miao, C. Zhang, and X. Li, "Monolithic barrier-all-around high electron mobility transistor with planar GaAs nanowire channel," Nano Lett., vol. 13, no. 6, pp. 2548-2552, Jun. 2013.

[13] C. Zhang and X. Li, "Planar GaAs nanowire tri-gate MOSFETs by vapor-liquid-solid growth," Solid-State Electron., vol. 93, pp. 40-42, Mar. 2014.

[14] C. Zhang, R. Dowdy, and X. Li, "High voltage gain MESFET amplifier using self-aligned MOCVD grown planar GaAs nanowires," in Proc. 71st Annu. Device Res. Conf. (DRC), Jun. 2013, pp. 63-64.

[15] H. Yamaguchi et al., "Excellent electric properties of free-standing InAs membranes," Appl. Phys. Lett., vol. 78, no. 16, pp. 2372-2374, 2001.

[16] Y. Song et al., "III-V junctionless gate-all-around nanowire MOSFETs for high linearity low power applications," IEEE Electron Device Lett., vol. 35, no. 3, pp. 324-326, Mar. 2014.

[17] X. Zhao et al., "Vertical nanowire InGaAs MOSFETs fabricated by a top-down approach," in IEDM Tech. Dig., Dec. 2013, pp. 695-698.

[18] A. W. Dey et al., "High-performance InAs nanowire MOSFETs," IEEE Electron Device Lett., vol. 33, no. 6, pp. 791-793, Jun. 2012.

[19] S. Birner et al., "nextnano: General purpose 3-D simulations," IEEE Trans. Electron Devices, vol. 54, no. 9, pp. 2137-2142, Sep. 2007.

[20] A. C. Ford et al., "Diameter-dependent electron mobility of InAs nanowires," Nano Lett., vol. 9, no. 1, pp. 360-365, 2009. 